\title{
Investigation of Phase Diagram and Bulk Thermodynamic Properties using PNJL Model with Eight-Quark Interactions
}

\author{
Abhijit Bhattacharyya* and Paramita Deb $\AA^{\Uparrow}$ \\ Department of Physics, University of Calcutta, \\ 92, A. P. C. Road, Kolkata - 700009, INDIA \\ Sanjay K. Ghosh \\ Department of Physics and Centre for Astroparticle Physics \& Space Science, \\ Bose Institute, 93/1, A. P. C Road, Kolkata - 700009, INDIA
}

\begin{abstract}
We present the bulk thermodynamic properties and phase diagram of strongly interacting matter in an extension of the 3-flavor NJL and PNJL models of QCD. Using a three momentum cut-off scheme, we have extended the multiquark interaction terms up to eight order so that the stability of the vacuum is ensured in these models. We explore the effects of various combinations of the two eight-quark couplings $g_{1}$ and $g_{2}$ and present a comparative study between the NJL and PNJL models as well as Lattice QCD data. The main effect of the eight-quark interaction term is to shift the critical end point in the $T-\mu$ phase diagram to a lower value of $\mu$ and higher value of $T$, thus bringing them closer to Lattice QCD results.
\end{abstract}

PACS numbers: 12.38.Aw, 12.38.Mh, 12.39.-x

\section{INTRODUCTION}

The strong interaction as described by Quantum Chromodynamics (QCD), is a remarkable branch of physics which shows a rich phase structure at finite temperature and density. At low temperatures and densities, the dominant degrees of freedom in our nature are color-singlet bound states of hadrons. However, due to the asymptotic freedom of QCD, it is expected that at very high temperatures and densities these hadrons break up to liberate quarks and gluons and form the quark-gluon plasma (QGP). The experimental exploration of such a phase transition from the confined hadronic phase to the deconfined QGP phase is being pursued actively in the Relativistic Heavy Ion Collider (RHIC) and more data are expected from Large Hadron Collider (LHC) running at CERN and in the future experiment at FAIR.

The transition region is however quite far from the asymptotic regime of QCD. This nonperturbative nature makes the study of these hot and dense matter quite non-trivial. The most reliable way to analyze the physics in this range of interest is to perform the numerical computation of a lattice version of the color SU(3) gauge theory (Lattice QCD/LQCD). The scheme is robust but numerically costly. Hence the most popular way to study the physics of the phase transition of strongly interacting matter is to look into one of the various possible effective models inspired from QCD. One of these is the Nambu-Jona-Lasinio (NJL) model [1-4] which incorporates the global symmetries of QCD quite nicely. A four quark interaction term in

\footnotetext{
*Electronic address: abphy@caluniv.ac.in

${ }^{\dagger}$ Electronic address: paramita.deb83@gmail.com

${ }^{\ddagger}$ Electronic address: sanjay@bosemain.boseinst.ac.in

§Electronic address: rajarshi@bosemain.boseinst.ac.in
} 
the NJL Lagrangian is able to generate the physics of spontaneous breaking of chiral symmetry - a property of QCD which is manifested as the nondegenerate chiral partners of the low-mass hadrons. But the major drawback of the NJL model is to have a reasonable description of the physics of color confinement. In this respect, the Polyakov loop extended NJL (PNJL) model [5] 8] tries to incorporate the fact that the chiral transition in QCD is of deconfining nature by introducing a background temporal gluon field.

In our previous work [9] we elaborately studied the $2+1$ flavor PNJL model [8], incorporating four-quark and six-quark interaction terms in the Lagrangian with three-momentum cut-off regularization. The six-quark interaction term (or the 't Hooft determinant term) preserves the $S U(3)_{L} \times S U(3)_{R}$ symmetry and breaks the $U(1)_{A}$ symmetry required due to the chiral anomaly. This term is also responsible for the flavor mixing in the $\eta_{0}$ and $\eta_{8}$ mesons to give $\eta$ and $\eta^{\prime}$ mesons.

However, due to the six-quark term the vacuum of the model becomes unbounded from below. This problem has become more obvious in the functional analysis of the Lagrangian. In the work of Reinhardt and Alkofer [10] the stationary phase approximation method was considered to bosonize the model and to calculate the contribution of the classical path at the lowest order. This lowest order results sums all tree diagrams in powers of the coupling constant of the six quark interaction. But the functional integral bosonization of the model shows several classical trajectories in that interval of the functional integration. Since at leading order there can be only one classical trajectory in the mean-field potential, the semiclassical potential derived from the functional integration differs from the mean-field one. So if we consider several classical trajectories, the effective potential of the theory gets unbounded from below.

In fact, the origin of multi-quark interactions is not very well-known. But we can find evidences of multi-quark interactions in the semi-classical theories based on QCD instanton vacuum [11]. There we can find certain correlations between two or more quarks by averaging over their positions and orientations in color space. Also in the instanton-gas model an infinite number of multi-quark interactions can be found beyond the zero mode approximation [12]. There are also some lattice measurements for the QCD vacuum which shows a hierarchy between the multi-quark interaction [13]. In this case the lowest four quark interaction term forms a stable vacuum by breaking the chiral symmetry spontaneously. But the next term in the hierarchy, the six quark interaction term, which is needed to mimic the $U_{A}(1)$ anomaly, destroys the ground state. So one cannot truncate the tower of multi-quark interactions at this level. The next candidate is the eight-quark interaction term which ensures the stability of the vacuum.

There are some recent findings [14 16] which show that the addition of the eight-quark interaction term in the Lagrangian may solve the problem of unstable vacuum. The $(2+1)$ flavor NJL model with an eight-quark interaction was studied in the four momentum cut-off and Pauli-Villars regularization scheme. The standard bosonization procedure of six and eightquark interactions was followed and the multi-quark vertices were replaced by purely mesonic ones by the stationary-phase method. Indeed the controversy between the mean-field approach and the functional integral approach has been removed by including the eight-quark interaction term in the Lagrangian, since it restricts the number of classical trajectories to one, giving a stable ground state of the system. Recently, Kashiwa et.al. [17, 18] have studied the chiral phase transition in the 2-flavor NJL and PNJL models with eight-quark interactions. The 2 -flavor model does not incorporate the six-quark interaction.

In this paper we first of all extend the $(2+1)$-flavor NJL and PNJL models to include the eightquark interactions at non-zero temperatures and densities. For this we use the 3 -momentum cut-off regularization scheme. Thereafter we thoroughly investigate the effect of the eight-quark term in the thermodynamic properties of strongly interacting matter.

We organize our paper as follows: in the next section we briefly describe the $2+1$ flavor PNJL 
model with eight-quark interaction term in the Lagrangian. For the Polyakov loop potential we incorporate Vandermonde term [7]. In sec. III we describe the thermodynamic potential of the PNJL model. We elaborately discuss the parameter sets chosen, and the stability criteria. The next two sections discuss the results of the paper, about the chiral phase transition and the bulk thermodynamic properties like pressure, trace anomaly at zero density and quark number density at finite temperature and density. We give a detailed analysis of the phase diagram and the critical end points at the next section. A summary of our results are available in the last section.

\section{THREE FLAVOR PNJL MODEL WITH EIGHT-QUARK INTERACTION}

In this section we briefly describe the formalism of the PNJL model with eight-quark interaction term. Some details on three flavor PNJL model with four and six quark interaction can be found in the literature [ $5,6,6,8,[9]$ and the NJL model with eight-quark interaction term in 14-16]. There is also some work on two flavor PNJL model with eight-quark interaction term 18].

In the PNJL model the gluon dynamics is reduced to the chiral point couplings between quarks and a simple temporal background gauge field which represents Polyakov Loop dynamics. The Polyakov line is represented as (see e.g. [19] and references therein),

$$
L(\bar{x})=\mathcal{P} \exp \left[i \int_{0}^{\beta} d \tau A_{4}(\bar{x}, \tau)\right]
$$

where $A_{4}=i A_{0}$ is the temporal component of Eucledian gauge field $\left(\bar{A}, A_{4}\right), \beta=\frac{1}{T}$, and $\mathcal{P}$ denotes path ordering. $L(\bar{x})$ transforms as a field with charge one under global Z(3) symmetry. The gluon dynamics can be described as an effective theory of the Polyakov loops [20]. The quark thermodynamics can be described by the NJL model [1]. Let us consider the $S U(3)_{f}$ version of PNJL model with eight-fermion interaction described by the Lagrangian,

$$
\begin{aligned}
\mathcal{L} & =\sum_{f=u, d, s} \bar{\psi}_{f} \gamma_{\mu} i D^{\mu} \psi_{f}-\sum_{f} m_{f} \bar{\psi}_{f} \psi_{f}+\sum_{f} \mu \gamma_{0} \bar{\psi}_{f} \psi_{f} \\
& +\frac{g_{S}}{2} \sum_{a=0, \ldots, 8}\left[\left(\bar{\psi} \lambda^{a} \psi\right)^{2}+\left(\bar{\psi} i \gamma_{5} \lambda^{a} \psi\right)^{2}\right]-g_{D}\left[\operatorname{det} \bar{\psi}_{f} P_{L} \psi_{f^{\prime}}+\operatorname{det} \bar{\psi}_{f} P_{R} \psi_{f^{\prime}}\right] \\
& +8 g_{1}\left[\left(\bar{\psi}_{i} P_{R} \psi_{m}\right)\left(\bar{\psi}_{m} P_{L} \psi_{i}\right)\right]^{2}+16 g_{2}\left[\left(\bar{\psi}_{i} P_{R} \psi_{m}\right)\left(\bar{\psi}_{m} P_{L} \psi_{j}\right)\left(\bar{\psi}_{j} P_{R} \psi_{k}\right)\left(\bar{\psi}_{k} P_{L} \psi_{i}\right)\right] \\
& -\mathcal{U}^{\prime}(\Phi[A], \bar{\Phi}[A], T) \\
& =\mathcal{L}_{0}+\mathcal{L}_{\mathcal{S B}}+\mathcal{L}_{\mu}+\mathcal{L}_{s}+\mathcal{L}_{\mathcal{K} \mathcal{M} \mathcal{T}}+\mathcal{L}_{8 q}^{1}+\mathcal{L}_{8 q}^{2}-\mathcal{U}^{\prime}
\end{aligned}
$$

where the matrices $P_{L, R}=\left(1 \pm \gamma_{5}\right) / 2$ are chiral projectors. In the above Lagrangian $\mathcal{L}_{0}$ is the Dirac term with gauge field interactions; $D^{\mu}=\partial^{\mu}-i A_{4} \delta_{\mu 4}$. $\mathcal{L}_{S B}$ is the mass term which breaks the symmetry explicitly. The mass of a particular flavor is denoted by $m_{f}$, where $f=u, d, s . \mathcal{L}_{s}$ is the four-fermi interaction term with coupling $g_{S}$. The next term, $\mathcal{L}_{K M T}$, which is a six-fermi interaction term, is invariant under $S U(3)_{L} \times S U(3)_{R}$ but breaks $U(1)_{A}$ symmetry. This term mimics the QCD chiral anomaly. The terms $\mathcal{L}_{8 q}^{1}$ and $\mathcal{L}_{8 q}^{2}$ are the eight-quark interaction terms which describe the spin zero interactions where $g_{1}$ and $g_{2}$ are the corresponding couplings. Here we have considered the interaction terms to be effectively local since the meson physics in the large $N_{c}$ limit is described by the local Lagrangian of this type. Since the coupling constants are dimensionful, the model is not renormalizable. So we have used three-momentum cut-off regulator $\Lambda$ to make quark loops finite. 
In earlier versions of the PNJL model we found that the Polyakov loop, which is the normalized trace of the Wilson line $L$, has become greater than 1 above $2 T_{C}[9,21,22]$. To solve this problem one has to take a proper Jacobian of transformation from the matrix valued field $L$ to $\Phi$ which will then constrain the value of $\Phi$ within 1 . One way to resolve this problem is to introduce Vandermonde term in the Polyakov loop potential [7]. Thus the potential $\mathcal{U}^{\prime}$ with the Vandermonde term can be expressed as

$$
\frac{\mathcal{U}^{\prime}(\Phi[A], \bar{\Phi}[A], T)}{T^{4}}=\frac{\mathcal{U}(\Phi[A], \bar{\Phi}[A], T)}{T^{4}}-\kappa \ln [J(\Phi, \bar{\Phi})]
$$

where $\mathcal{U}(\phi)$ is the Landau-Ginzburg type potential given by [6],

$$
\frac{\mathcal{U}(\Phi, \bar{\Phi}, T)}{T^{4}}=-\frac{b_{2}(T)}{2} \bar{\Phi} \Phi-\frac{b_{3}}{6}\left(\Phi^{3}+\bar{\Phi}^{3}\right)+\frac{b_{4}}{4}(\bar{\Phi} \Phi)^{2}
$$

with

$$
\begin{array}{r}
\Phi=\left(\operatorname{Tr}_{c} L\right) / N_{c}, \quad \bar{\Phi}=\left(\operatorname{Tr}_{c} L^{\dagger}\right) / N_{c} \\
b_{2}(T)=a_{0}+a_{1}\left(\frac{T_{0}}{T}\right)+a_{2}\left(\frac{T_{0}}{T}\right)^{2}+a_{3}\left(\frac{T_{0}}{T}\right)^{3},
\end{array}
$$

$b_{3}$ and $b_{4}$ being constants. The second term in eqn. 3 is known as Vandermonde term, where $J(\Phi, \bar{\Phi})$ is the Jacobian of transformation from Wilson line $L$ to $(\Phi, \bar{\Phi})$ written as

$$
J[\Phi, \bar{\Phi}]=\left(27 / 24 \pi^{2}\right)\left(1-6 \Phi \bar{\Phi}+4\left(\Phi^{3}+\bar{\Phi}^{3}\right)-3(\Phi \bar{\Phi})^{2}\right.
$$

$J(\Phi, \bar{\Phi})$ is also known as Vandermonde determinant and is not explicitly space time dependent. The value of the dimensionless parameter $\kappa$ will be determined phenomenologically. The coefficient $\kappa$ in the VDM term can in general have some temperature and/or chemical potential dependence. But here we take a constant value in such a way that we can get the pressure and the transition temperature as close as possible to the lattice QCD results for the PNJL model with and without the eight-quark interaction. This coefficient has been tabulated later.

In order to study the chiral transition of the system we consider the Mean Field Approximation (MFA) of the eqn. (2) to get the field equations for $\Phi, \bar{\Phi}, \sigma$, where it is assumed that the system is described as an assembly of non-interacting particles moving in the mean field. The theory is analogous to the BCS theory of superconductor, where the pairing of two electrons leads to the condensation causing the gap in the energy spectra. Similarly in this model, due to the dynamical breaking of $S U(3)_{L} \times S U(3)_{R}$ symmetry to $S U(3)_{V}$ a composite operator picks up a nonzero vacuum expectation value leading to $\langle\bar{\psi} \psi\rangle$ condensation. Due to the dynamical breaking of chiral symmetry, nine goldstone bosons appear for $N_{f}=3$ model. The quark condensate is given as,

$$
\left\langle\bar{\psi}_{f} \psi_{f}\right\rangle=-i N_{c} \mathcal{L} t_{y \rightarrow x^{+}}\left(\operatorname{tr} S_{f}(x-y)\right)
$$

where trace is over color and spin states. The self-consistent gap equation for the constituent masses are,

$$
M_{f}=m_{f}-2 g_{S} \sigma_{f}+\frac{g_{D}}{2} \sigma_{f+1} \sigma_{f+2}-2 g_{1} \sigma_{f}\left(\sigma_{u}^{2}+\sigma_{d}^{2}+\sigma_{s}^{2}\right)-4 g_{2} \sigma_{f}^{3}
$$

here $\sigma_{f}=\left\langle\bar{\psi}_{f} \psi_{f}\right\rangle$ denotes chiral condensate of the quark with flavor $f . f$ denotes the flavor $u, d, s$ respectively. Here if we consider $\sigma_{f}=\sigma_{u}$, then $\sigma_{f+1}=\sigma_{d}$ and $\sigma_{f+2}=\sigma_{s}$. Similarly 
if $\sigma_{f}=\sigma_{d}$ then $\sigma_{f+1}=\sigma_{s}$ and $\sigma_{f+2}=\sigma_{d}$, if $\sigma_{f}=\sigma_{s}$ then $\sigma_{f+1}=\sigma_{u}$ and $\sigma_{f+2}=\sigma_{d}$. The expression for $\sigma_{f}$ at $T=0$ and $\mu=0$ can be written as [8]

$$
\sigma_{f}=-\frac{3 M_{f}}{\pi^{2}} \int_{0}^{\Lambda} \frac{p^{2}}{\sqrt{p^{2}+M_{f}^{2}}} d p
$$

$\Lambda$ being the three-momentum cut off.

\section{THERMODYNAMIC POTENTIAL AND PARAMETER FITTING}

The primary aim of our work is to study the thermodynamic properties of the strongly interacting matter using the PNJL model with eight-quark interactions at zero and finite chemical potentials. To do so we now need to fix the parameters in both the NJL and Polyakov loop potentials. The thermodynamic potential for the multi-fermion interaction in MFA of the PNJL model can be written as

$$
\begin{aligned}
\Omega(\Phi, \bar{\Phi}, M, T, \mu) & =\mathcal{U}^{\prime}[\Phi, \bar{\Phi}, T]+2 g_{S} \sum_{f=u, d, s} \sigma_{f}^{2}-\frac{g_{D}}{2} \sigma_{u} \sigma_{d} \sigma_{s}+3 \frac{g_{1}}{2}\left(\sigma_{f}^{2}\right)^{2}+3 g_{2} \sigma_{f}^{4} \\
& -T \sum_{n} \int \frac{d^{3} p}{(2 \pi)^{3}} \operatorname{Tr} \ln \frac{S^{-1}\left(i \omega_{n}, \bar{p}\right)}{T}
\end{aligned}
$$

where $\omega_{n}=\pi T(2 n+1)$ are Matsubara frequencies for fermions. The inverse quark propagator is given in momentum space by

$$
S^{-1}=\gamma_{0}\left(p^{0}+\mu-i A_{4}\right)-\vec{\gamma} \cdot \vec{p}-M
$$

using the identity $\operatorname{Tr} \ln (X)=\ln \operatorname{det}(X)$, we get

$$
\begin{aligned}
\Omega & =\mathcal{U}^{\prime}[\Phi, \bar{\Phi}, T]+2 g_{S} \sum_{f=u, d, s} \sigma_{f}^{2}-\frac{g_{D}}{2} \sigma_{u} \sigma_{d} \sigma_{s}+3 \frac{g_{1}}{2}\left(\sigma_{f}^{2}\right)^{2} \\
& +3 g_{2} \sigma_{f}^{4}-6 \sum_{f} \int_{0}^{\Lambda} \frac{d^{3} p}{(2 \pi)^{3}} E_{p f} \Theta(\Lambda-|\vec{p}|) \\
& -2 \sum_{f} T \int_{0}^{\infty} \frac{d^{3} p}{(2 \pi)^{3}} \ln \left[1+3\left(\Phi+\bar{\Phi} e^{\frac{-\left(E_{p f}-\mu\right)}{T}}\right) e^{\frac{-\left(E_{p f}-\mu\right)}{T}}+e^{\frac{-3\left(E_{p f}-\mu\right)}{T}}\right] \\
& -2 \sum_{f} T \int_{0}^{\infty} \frac{d^{3} p}{(2 \pi)^{3}} \ln \left[1+3\left(\bar{\Phi}+\Phi e^{\frac{-\left(E_{p f}+\mu\right)}{T}}\right) e^{\frac{-\left(E_{p f}+\mu\right)}{T}}+e^{\frac{-3\left(E_{p f}+\mu\right)}{T}}\right]
\end{aligned}
$$

where $E_{p f}=\sqrt{p^{2}+M_{f}^{2}}$ is the single quasiparticle energy, $\sigma_{f}^{2}=\left(\sigma_{u}^{2}+\sigma_{d}^{2}+\sigma_{s}^{2}\right)$ and $\sigma_{f}^{4}=$ $\left(\sigma_{u}^{4}+\sigma_{d}^{4}+\sigma_{s}^{4}\right)$. In the above integrals, the vacuum integral has a cutoff $\Lambda$ whereas the medium dependent integrals have been extended to infinity. The main idea to incorporate the eightquark interaction term in the Lagrangian is to stabilize the vacuum. The detailed discussion of the stability criteria of the vacuum can be found in the literature [14, 15].

The parameters of the NJL part of the Lagrangian are the current quark masses $m_{u}, m_{d}$ and $m_{s}$, the coupling constants $g_{s}, g_{D}, g_{1}$ and $g_{2}$ and the three-momentum cutoff $\Lambda$ characterizing 


\begin{tabular}{|c|c|c|c|c|c|c|c|c|}
\hline \multicolumn{7}{|c|}{ Sets } & Physical \\
Parameter \\
\cline { 1 - 1 } six-quark & \multicolumn{3}{|c|}{ eight-quark } & values \\
\hline$m_{\pi}$ & $m_{\pi}$ & $m_{\pi}$ & $m_{\pi}$ & $m_{\pi}$ & $m_{\pi}$ & $m_{\pi}$ & $m_{\pi}$ & $m_{\pi}=138 \mathrm{MeV}$ \\
$f_{\pi}$ & $f_{\pi}$ & $f_{\pi}$ & $f_{\pi}$ & $f_{\pi}$ & $f_{\pi}$ & $f_{\pi}$ & $f_{\pi}$ & $f_{\pi}=93 \mathrm{MeV}$ \\
$m_{k}$ & $m_{k}$ & $m_{k}$ & $m_{k}$ & $m_{k}$ & $m_{k}$ & $m_{k}$ & $m_{k}$ & $m_{k}=494 \mathrm{MeV}$ \\
- & - & - & - & $f_{k}$ & $f_{k}$ & $f_{k}$ & $f_{k}$ & $f_{k}=117 \mathrm{MeV}$ \\
$m_{\eta}$ & $m_{\eta}$ & $m_{\eta}$ & - & $m_{\eta}$ & $m_{\eta}$ & $m_{\eta}$ & - & $m_{\eta}=480 \mathrm{MeV}$ \\
- & - & - & - & $m_{\eta^{\prime}}$ & $m_{\eta^{\prime}}$ & $m_{\eta^{\prime}}$ & $m_{\eta^{\prime}}$ & $m_{\eta^{\prime}}=957 \mathrm{MeV}$ \\
- & $m_{\sigma}$ & - & $m_{\sigma}$ & - & $m_{\sigma}$ & - & $m_{\sigma}$ & $m_{\sigma}=680 \mathrm{MeV}$ \\
$m_{u}$ & - & - & $m_{u}$ & $m_{u}$ & - & - & $m_{u}$ & $m_{u}=5.5 \mathrm{MeV}$ \\
- & - & $\sigma_{u}$ & - & - & - & $\sigma_{u}$ & - & $\sigma_{u}^{1 / 3}=-248 \mathrm{MeV}$ \\
\hline
\end{tabular}

TABLE I: (Color online) The PNJL model parameters are fitted to reproduce the different physical parameters as indicated above.

\begin{tabular}{|c|c|c|c|c|c|c|c|c|c|c|}
\hline Set & $\begin{array}{c}m_{u} \\
\mathrm{MeV}\end{array}$ & $\begin{array}{c}m_{s} \\
\mathrm{MeV}\end{array}$ & $\begin{array}{c}\Lambda \\
\mathrm{MeV}\end{array}$ & $g_{S} \Lambda^{2}$ & $g_{D} \Lambda^{5}$ & $\begin{array}{c}g_{1} \times 10^{-21} \\
\mathrm{MeV}^{-8}\end{array}$ & $\begin{array}{c}g_{2} \times 10^{-22} \\
\mathrm{MeV}^{-8}\end{array}$ & $\kappa$ & $\begin{array}{c}T_{C}^{\text {PJL }} \\
\mathrm{MeV}\end{array}$ & $\begin{array}{c}T_{C}^{N J L} \\
\mathrm{MeV}\end{array}$ \\
\hline$a$ & 5.5 & 134.758 & 631.357 & 3.664 & 74.636 & 0.0 & 0.0 & .13 & 181.0 & 170.55 \\
$b$ & 5.406 & 133.227 & 641.357 & 3.717 & 61.309 & 0.0 & 0.0 & .18 & 182.2 & 170.25 \\
$c$ & 5.418 & 133.562 & 640.206 & 3.637 & 70.849 & 0.0 & 0.0 & .14 & 180.5 & 169.25 \\
$d$ & 5.5 & 133.532 & 631.337 & 4.229 & 14.461 & 0.0 & 0.0 & .11 & 183.5 & 176.65 \\
$e$ & 5.5 & 183.468 & 637.720 & 2.914 & 75.968 & 2.193 & -5.890 & .06 & 168.5 & 141.35 \\
$f$ & 12.509 & 181.863 & 628.933 & 2.986 & 75.444 & 2.007 & -4.538 & .07 & 171.8 & 150.05 \\
$g$ & 8.742 & 179.498 & 640.206 & 2.928 & 75.382 & 1.929 & -4.840 & .05 & 169.0 & 144.25 \\
$h$ & 5.5 & 187.786 & 628.933 & 2.956 & 75.983 & 2.425 & -6.445 & .08 & 170.5 & 144.15 \\
\hline
\end{tabular}

TABLE II: (Color online) Parameters of the $S U(3)$ NJL part and different value of $T_{C}$ at $\mu=0$ and the value of $\kappa$ for all input parameter sets.

the scale of the chiral symmetry breaking. Here we consider the isospin symmetric limit $m_{u}=$ $m_{d}$. These parameters are determined by reproducing few physical quantities like pion mass $m_{\pi}=138 \mathrm{MeV}$, kaon mass $m_{K}=494 \mathrm{MeV}$, eta mass $m_{\eta}=480 \mathrm{MeV}$, eta prime mass $m_{\eta}^{\prime}=$ $957 \mathrm{MeV}$, pion decay constant $f_{\pi}=93 \mathrm{MeV}$, kaon decay constant $f_{K}=117 \mathrm{MeV}$, u quark condensate $\sigma_{u}^{1 / 3}=-248 \mathrm{MeV}$ and/or the mass of sigma meson $m_{\sigma}=680 \mathrm{MeV}$.

We consider two cases of up to six-quark $\left(g_{1}=0=g_{2}\right)$ and up to eight-quark $\left(g_{1} \neq 0 \neq g_{2}\right)$ interactions each with four sets $(a, b, c, d)$ and $(e, f, g, h)$ respectively. In table \ we have tabulated different physical observables and their values for the different sets. In set $(a, d)$ and $(e, h) m_{u}$ is kept fixed at $5.5 \mathrm{MeV}$ at $1 \mathrm{GeV}$ scale since the values determined from the evaluation of the current matrix elements at low energies are also centered around $5.5 \mathrm{MeV}$. In sets $b$ and $f$ the mass of sigma meson is considered as fixed to obtain the model parameters whereas in sets $c$ and $g$ the u quark condensate is considered. In sets $(b, c)$ we fit the parameters $\left(m_{u}, m_{s}, \Lambda, g_{S}, g_{D}\right)$ by fixing $\left(m_{\pi}, m_{K}, m_{\eta}, f_{\pi}\right.$ and $m_{\sigma}$ or $\left.\sigma_{u}\right)$ and in sets $(f, g)$ the parameters $\left(m_{u}, m_{s}, \Lambda, g_{S}, g_{D}, g_{1}, g_{2}\right)$ are obtained by fixing $\left(m_{\pi}, m_{K}, m_{\eta}, m_{\eta}^{\prime}, f_{\pi}, f_{K}\right.$ and $m_{\sigma}$ or $\left.\sigma_{u}\right)$. How- 
ever in set $d$ and $h$ instead of $m_{\eta}$ we have used $m_{\sigma}=680 \mathrm{MeV}$ for fitting the same parameters as $m_{\sigma}$ is the most sensitive to the eight-quark couplings.

The sensitivity of sigma mass to the eight-quark coupling constant have been also discussed in the paper [15], however instead of producing the eight-quark couplings, they put the values of these coupling constants by hand. The reason behind producing four sets of parameters is to show the effect of different physical channels on the different parameters. We would like to point out here that the mass of the sigma meson is taken as $680 \mathrm{MeV}$ to fit the parameters in sets $b$ and $f$, due to which we obtain $m_{u}=12.5 \mathrm{MeV}$ in set $f$. The value of $m_{u}$ in set $f$ is higher than the value of $m_{u}=5-9 \mathrm{MeV}$ that is usually quoted in the literature. A lower mass value of sigma meson $\sim 660 \mathrm{MeV}$ may give a lower value of current $\mathrm{u}$ quark mass but then the constituent $\mathrm{u}$ quark mass will be much higher than the value obtained for other input parameter sets. In our analysis we will consider the plots for the input parameter sets in the following combinations:

- Set 1 : Sets $(a, e)$ (six-quark,eight-quark). Here $m_{u}=5.5 \mathrm{MeV}$ is held fixed.

- Set 2 : Sets $(b, f)$ (six-quark,eight-quark). Here $m_{\sigma}=680 \mathrm{MeV}$ is used to fit parameters.

- Set 3 : Sets $(c, g)$ (six-quark,eight-quark). Here $\sigma_{u}$ is used instead of $m_{\sigma}$.

- Set 4 : Sets $(d, h)$ (six-quark,eight-quark). Here $m_{u}=5.5 \mathrm{MeV}$ is held fixed and $m_{\sigma}=$ $680 \mathrm{MeV}$ is used to fit parameters.

The resulting model parameter values of the NJL part at zero temperature, obtained in our fit is given in the columns $2-8$ of table

For the Polyakov loop part we have to consider the finite temperature behavior of the PNJL model. As discussed in [7] we are able to tune the dimensionless coupling $\kappa$ in the Vandermonde term and obtain reasonable behavior of the mean fields. We thus choose the following set of parameters,

$$
a_{0}=6.75, a_{1}=-1.95, a_{2}=2.625 a_{3}=-7.44, b_{3}=0.75, b_{4}=7.5, T_{0}=190 \mathrm{MeV}
$$

The remaining parameter is the dimensionless coupling $\kappa$ in the Vandermonde term which has been tabulated in column 9 of table $[1$. This has been obtained by choosing suitable value of $\kappa$ so that the pressure in PNJL model follows the Lattice QCD data as closely as possible.

Our job is now to estimate the transition temperatures at different quark chemical potentials. In order to obtain this we search for the minimum of the thermodynamic potential which gives the temperature and density dependence of the fields. The point of inflection or the gap, as the case may be, of these fields give the transition temperature and chemical potential. This tusk has been carried out for both NJL and PNJL models. The transition temperatures at zero chemical potential have been tabulated in the last two columns of table II. The most important observation in this regard is that the introduction of the eight quark interaction lowers the transition temperature to the range $150-190 \mathrm{MeV}$.

The pressure of the strongly interacting matter obtained from our expression of the thermodynamic potential is,

$$
P(T, \mu)=-\Omega(T, \mu)
$$

where $T$ is the temperature and $\mu$ is the quark chemical potential. In fig. 1 we show the variation of the scaled pressure $P / P_{S B}$ with $T / T_{C}$ in both the NJL and PNJL models for up to six-quark as well as up to eight-quark interactions. The plots show that the pressure is slightly smaller near the transition region due to eight-quark interaction term. In general, the shift of 

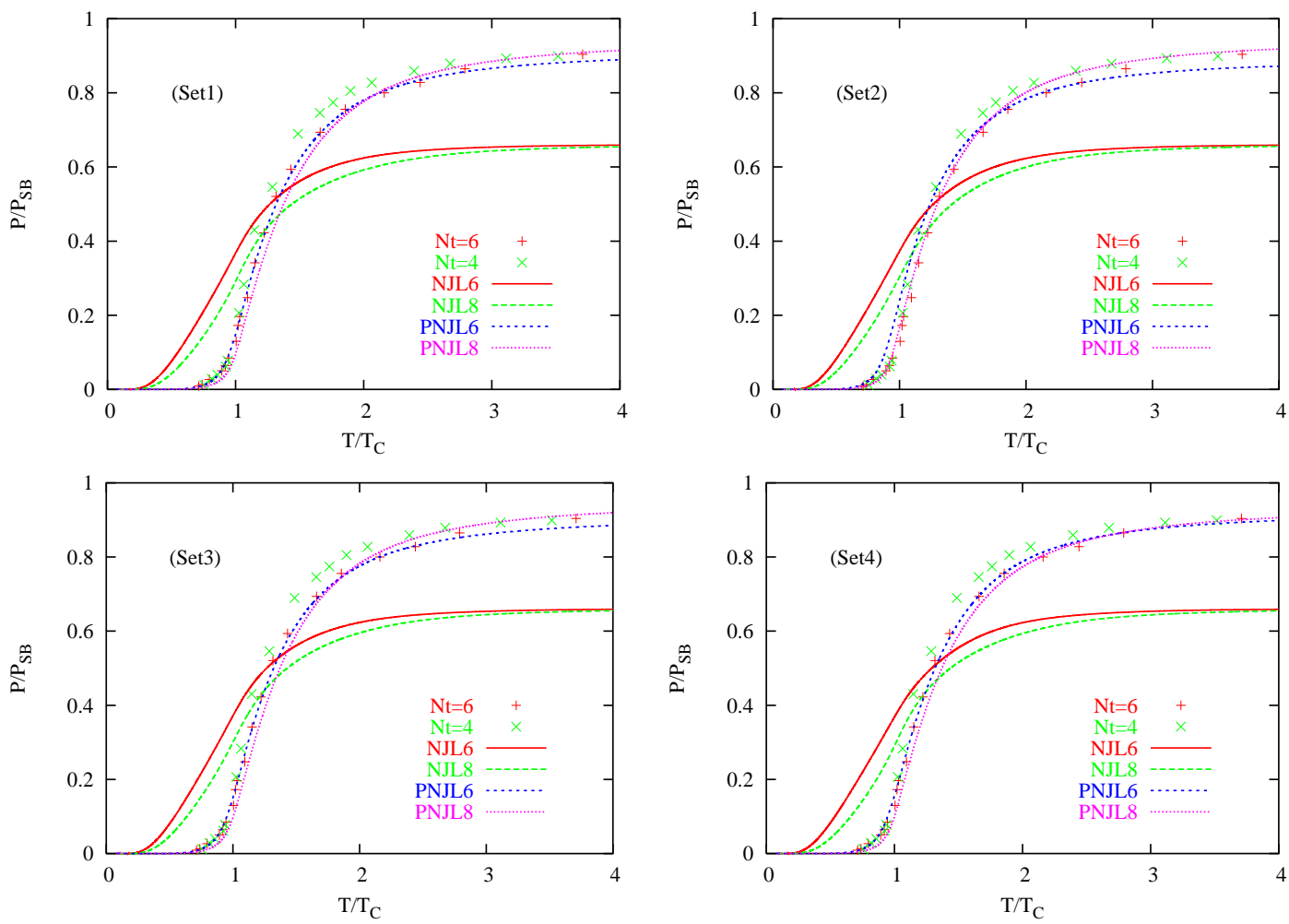

FIG. 1: (Color online) Variation of $P / P_{S B}$ with $T / T_{C}$, at $\mu=0$ for both NJL and PNJL model. For details of the sets see text in section III

pressure in the whole range of temperature ranges from $10 \%-20 \%$. We have also compared our results with those of the lattice QCD data 23] with temporal extent $N \tau=4$ and $N \tau=6$. In fact the pressure in the PNJL model was obtained to give the best possible fit to the lattice data with $N \tau=6$. We find that for all input parameter sets it is possible to have an impressive similarity with the results of the lattice data. On the other hand the results for the NJL model differ considerably from the lattice data.

Another basic thermodynamic quantity is the energy-momentum tensor. The thermal contribution of the trace of this energy-momentum tensor can be defined by the difference of $\epsilon$ and $3 p$, where $\epsilon$ is the energy density and $p$ is the pressure. So in thermodynamics the traceless energy-momentum tensor means $\Theta_{\mu \mu}=(\epsilon-3 p)=0$. The trace of the energy momentum tensor is vanishing at the classical level when the theory has no mass scale. We know that QCD in the chiral limit is scale invariant, which means that in massless QCD, $\Theta_{\mu \mu}$ is zero unless quantum corrections are taken into account. Thus in a conformally symmetric theory, for example a theory of free massless gluons, $\Theta_{\mu \mu}=0$. Therefore this quantity measures the breaking of conformal symmetry in the interaction theory. It is straightforward to evaluate the $(\epsilon-3 p)$ or the interaction measure from the thermodynamic potential and pressure as follows:

$$
(\epsilon-3 p) / T^{4}=T \frac{\partial\left(p / T^{4}\right)}{\partial T}
$$

In fig. 2 we show the variation of the $(\epsilon-3 p) / T^{4}$ with temperature for both the models 

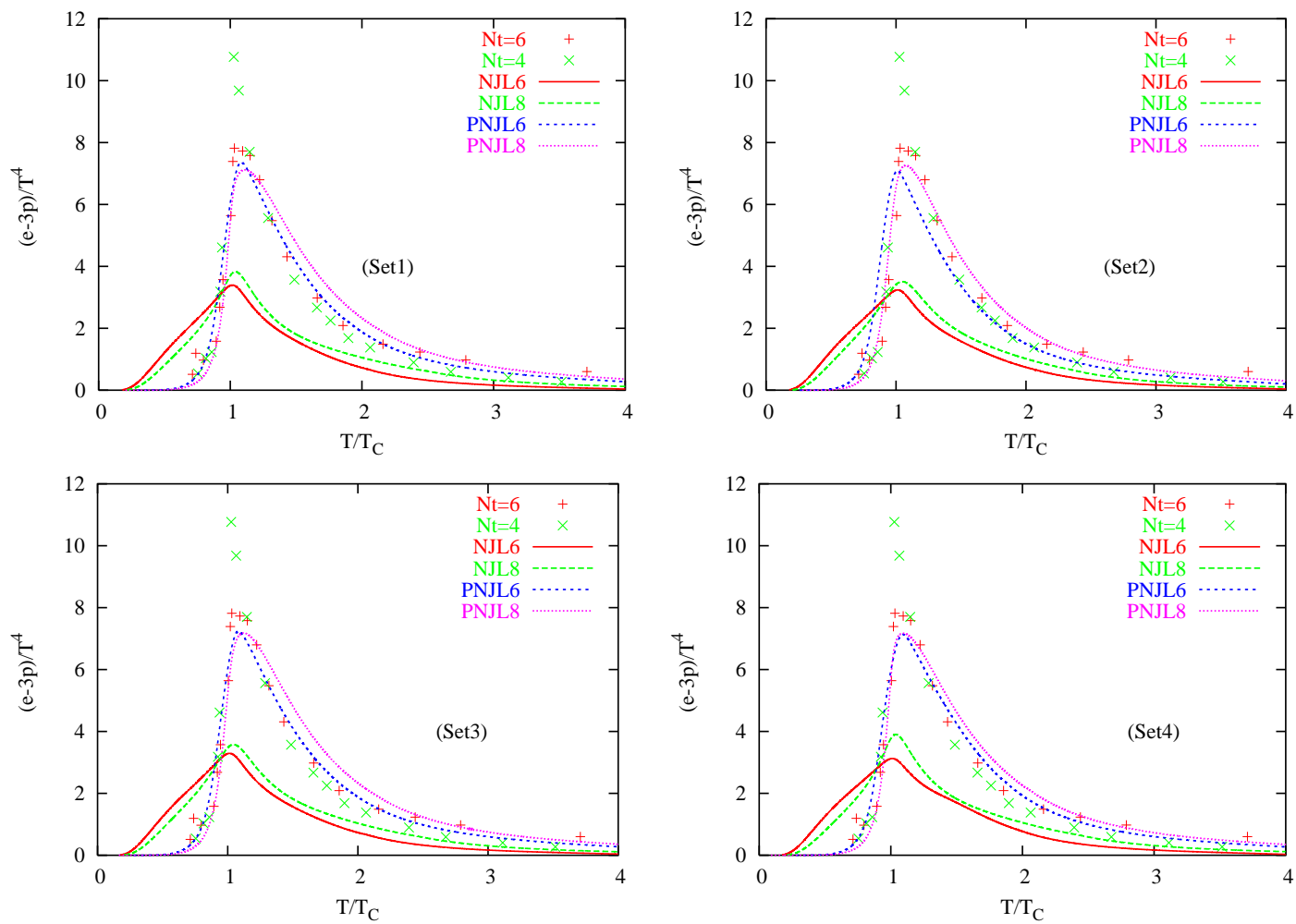

FIG. 2: (Color online) Variation of $(\epsilon-3 p) / T^{4}$ with $T / T_{C}$, at $\mu=0$ for both NJL and PNJL model. For details of the sets see text in section III

with and without eight-quark interaction. At temperatures just above $T_{C}$ the plots show a peak due to the largest deviations of $(\epsilon-3 p)$ from the conformal limit, $\epsilon=3 p$. These peaks establish a prominent structure of trace anomaly which is consistent with lattice calculations. The introduction of finite value of $g_{1}$ and $g_{2}$ slightly lowers the peak position in PNJL model which ensures reduction of trace anomaly due to the eight-quark interaction term. At high temperatures the trace anomaly dropped rapidly similar to that obtained from the lattice data. From the plots we can see that in this temperature region much larger than $T_{C}$, the trace anomaly drop less rapidly for plots with finite value of $g_{1}$ and $g_{2}$ than the plots with $g_{1}=g_{2}=0$. In all diagrams the peak position for $N_{\tau}=4$ result is much higher than the $N_{\tau}=6$ and the peak positions of the $(\epsilon-3 p) / T^{4}$ for the PNJL model are slightly lower than the peak position of the lattice result for $N_{\tau}=6$. It remains to be seen if this is close to the continuum limit of lattice data. At low temperature region the plots for all the parameter sets coincide very well with the lattice data, but at high temperature the parameter sets with the six-quark interaction are in better agreement with the lattice data. However the deviation of the plots with the eight-quark interaction term from the lattice results are also very small and a continuum extrapolation can be expected to yield even a better agreement. In pure gauge theory, it has been noted that $(\epsilon-3 p)$ is quite significant above the deconfinement temperature [24] and $\sim T^{2}$ for temperatures up to a few times the deconfinement temperature [25]. A similar behavior can be found in the PNJL model. 

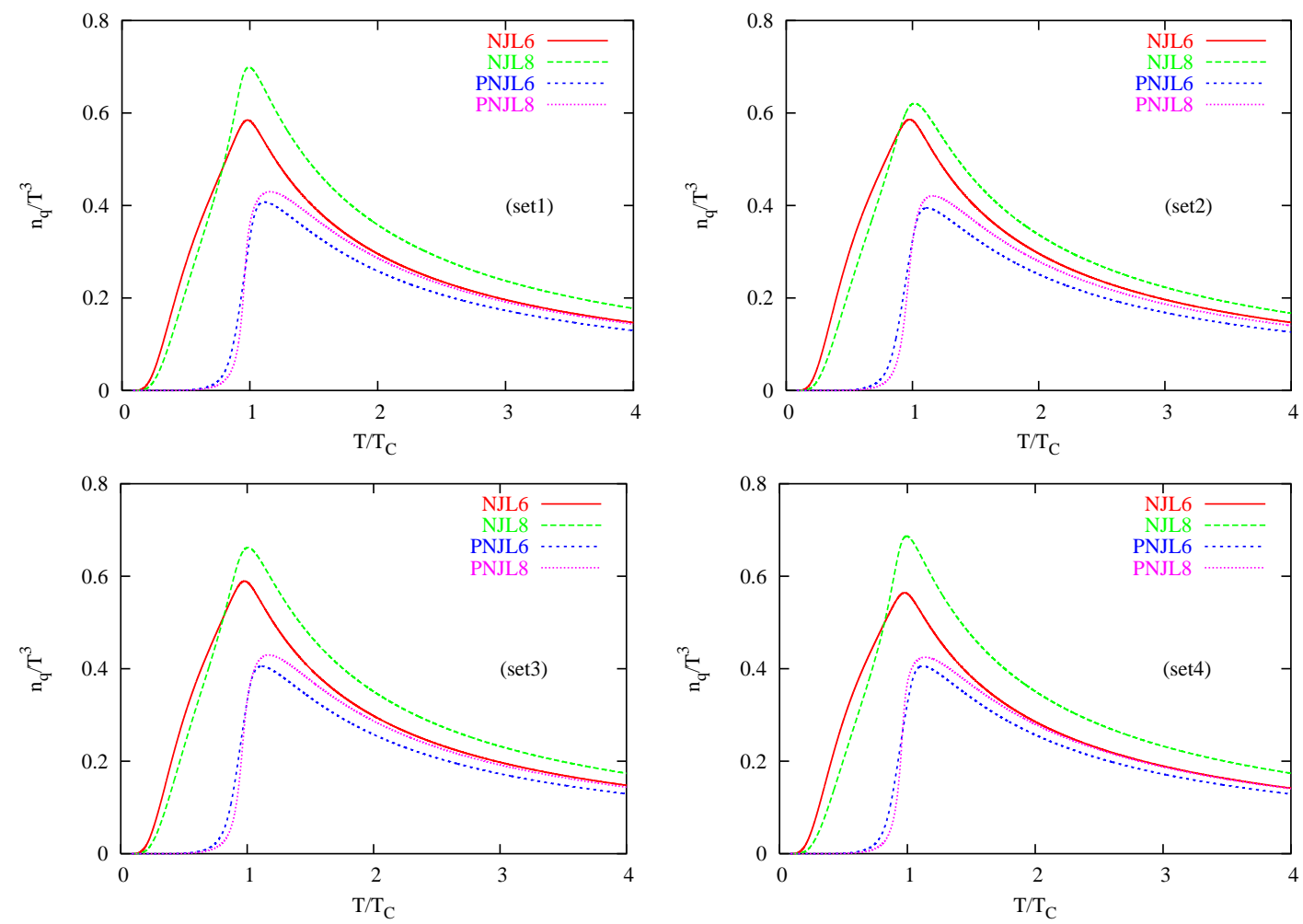

FIG. 3: (Color online) Variation of $n_{q} / T^{3}$ with temperature, at $\mu=100 \mathrm{MeV}$ for both NJL and PNJL models. For details of the sets see text in section [II.

The scaled quark number density is defined as:

$$
\frac{n_{q}(T, \mu)}{T^{3}}=-\frac{1}{T^{3}} \frac{\partial \Omega(T, \mu)}{\partial \mu}
$$

We have plotted the quark number density as a function of $T / T_{c}$ at $\mu=100 \mathrm{MeV}$ for both the models in fig. 3 and studied the effect of eight-quark interaction. It can be seen that at fixed values of temperature and chemical potential the $n_{q}$ in PNJL model is much lower than that in the NJL model below $T_{c}$. This is evidently an effect of confinement in the PNJL model. At a fixed quark chemical potential the quark number density for the PNJL model is almost vanishing below the chiral transition temperature, but rises very quickly in the vicinity of the transition temperature. However in case of the NJL model the rise in the quark number density starts much below the transition temperature. Interestingly, the addition of the eight-quark interaction term raises the quark number significantly above $T_{c}$ for all cases at hand.

\section{PHASE DIAGRAMS AND CRITICAL END POINT}

The position of the critical endpoint (CEP) in the phase diagram is one of the most interesting issue of the hot and dense strongly interacting matter. The phase diagrams are usually 

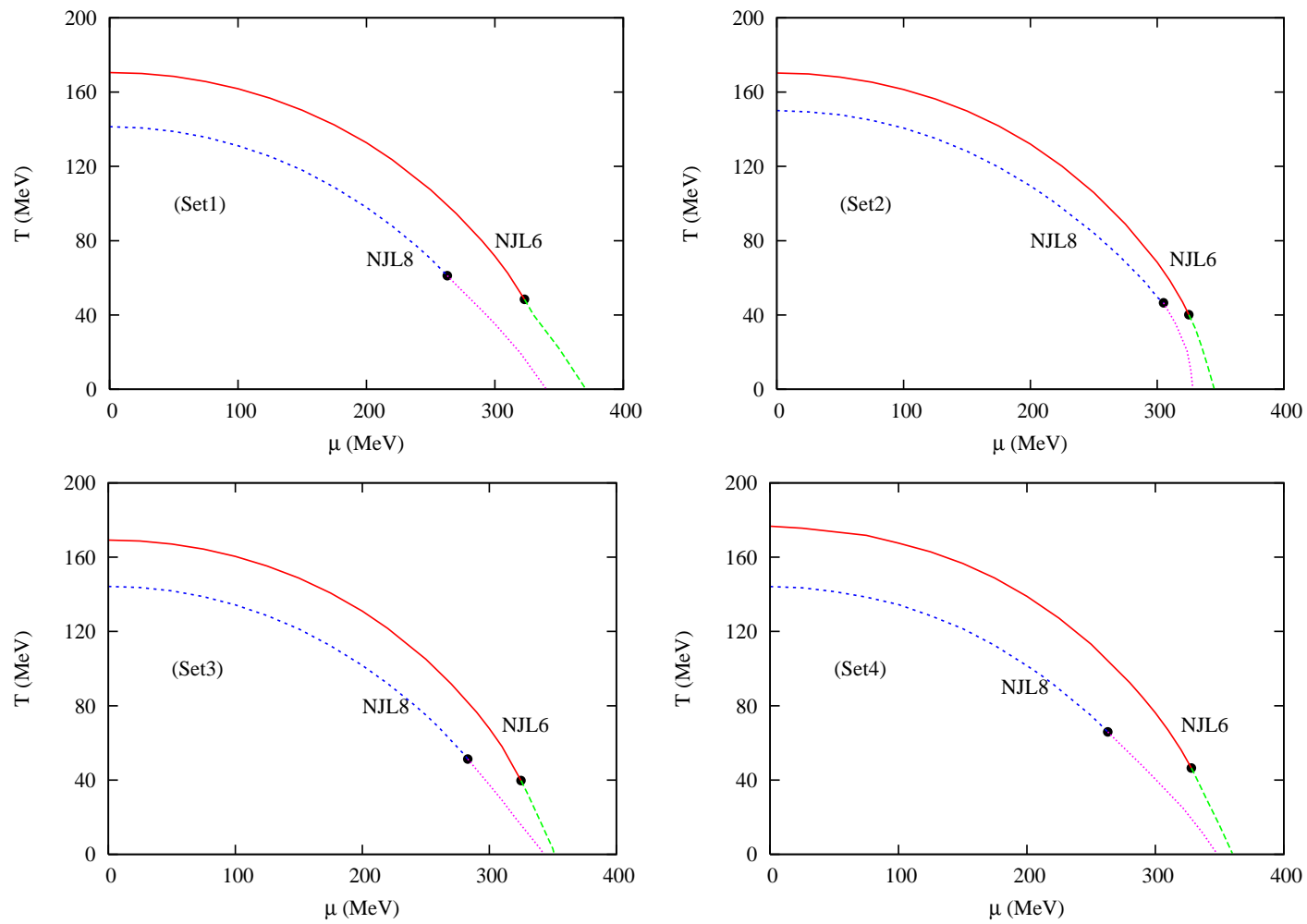

FIG. 4: (Color online) Phase diagram in $\mu$ with $T$ for NJL model. For details of the sets see text in section III.

obtained by identifying the critical temperature with the temperature at which the light quark chiral condensate has a jump (first order) or from the maximal point of the derivative of light quark condensate with respect to the temperature for different chemical potentials. CEP is the point which separate the cross-over transition from the first order phase transition. There have been a number of studies in lattice QCD to find out the CEP in the $T-\mu$ diagram [26] and also in QCD inspired models. In three flavor NJL model with the parameters of Hatsuda-Kunihiro [3] the location of CEP is found to be $\left(T_{C}, \mu_{C}\right)=(48 \mathrm{MeV}, 324 \mathrm{MeV})$, however in our previous work with three flavor PNJL model we have shown the position of CEP at $\left(T_{C}, \mu_{C}\right)=(92 \mathrm{MeV}, 314 \mathrm{MeV})$ which establishes the fact that the location of the CEP goes up in temperature in the PNJL model [9]. The reason behind this shift to the higher value of temperature is due to the suppression of quark excitations at finite temperature and density by the Polyakov loop. However the lattice estimates of the CEP vary cosiderably between different groups and the value of $\mu_{C}(\leq 150 \mathrm{MeV})$ tend to be much lower than the PNJL value. In this paper we try to develop a comparative study with our four input parameter sets for both NJL and PNJL model with and without eight-quark interaction. In fig. 4 and fig. 5 we have shown the phase diagrams of NJL and PNJL model with and without eight-quark interaction term for our four input parameter sets. In the region $T$ less than $T_{C}$ and $\mu$ greater than $\mu_{C}$ the chiral and deconfinement transitions are first order and occur almost at same $T$ and $\mu$. This can be realized from the generalized Clausius-Clapeyron relation for the system with multiple order parameters, which shows that in case of first order phase transition all discontinuities appear 

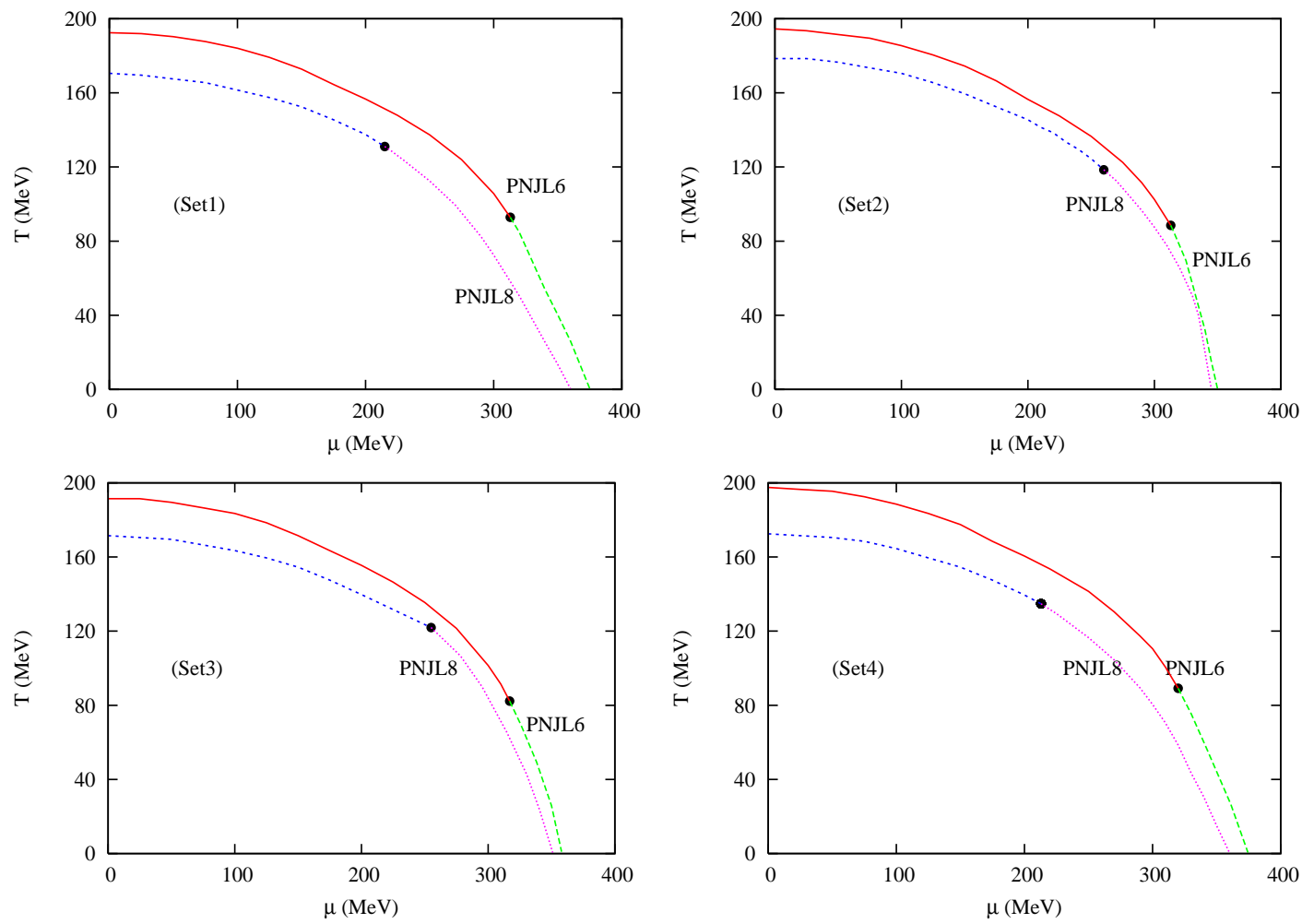

FIG. 5: (Color online) Phase diagram in $\mu$ with $T$ for PNJL model. For details of the sets see text in section III.

at same $T$ and $\mu$. In table III we have shown the values of CEP for different sets of parameters in both NJL and PNJL model. From the parameter sets we can clearly infer that the newly added eight-quark interaction term shifts the CEP towards lower $\mu$ and higher $T$ value for both NJL and PNJL model. The recent work in lattice QCD predicts that the possible region where CEP should exist is $\mu_{C} / T_{C} \leq 2.5$ [27]. From the table III we can observe that the PNJL model with eight-quark interaction term only satisfy this criteria. For the PNJL model without eight-quark interaction term this ratio becomes greater than 3 for all parameter sets. Moreover in case of NJL model with eight-quark interaction term, this ratio is not within the allowed region. This feature establishes the importance of including the eight-quark interaction term in the Lagrangian.

\section{CONCLUSION}

To conclude, we have studied the bulk thermodynamic properties and the phase diagram of the PNJL and NJL model with eight-quark interaction. This is the first time that 3-flavor PNJL model has been studied with eight-quark interaction and three-momentum cutoff.

We have used different sets of physical observables to fix the input parameters for our model (including the eight quark couplings). In particular, we can observe the sensitivity of the sigma 


\begin{tabular}{|c|c|c|c|c|}
\hline \multirow{2}{*}{ Model Index } & \multicolumn{4}{|c|}{$\left(\mu_{C} \mathrm{MeV}, \mathrm{T}_{\mathrm{c}} \mathrm{MeV}\right)$} \\
\cline { 2 - 5 } & set 1 & set 2 & set 3 & set 4 \\
\hline NJL (6- quark) & $(323,48.5)$ & $(325,40.15)$ & $(325,39.8)$ & $(328,46.45)$ \\
NJL (8 - quark) & $(263,61.2)$ & $(305,46.55)$ & $(283,51.4)$ & $(263,65.95)$ \\
PNJL (6 - quark) & $(313,92.85)$ & $(313,88.5)$ & $(317,82.25)$ & $(320,89.15)$ \\
PNJL (8 - quark) & $(260,118.5)$ & $(237,122.05)$ & $(255,121.95)$ & $(213,134.75)$ \\
\hline
\end{tabular}

TABLE III: (Color online) The values of $\left(\mu_{C}, T_{C}\right)$ for different sets of NJL and PNJL model

mass to the eight-quark coupling constants. However the value of sigma mass $m_{\sigma}=680 \mathrm{MeV}$ we took as one of the variable for fitting the input parameters with eight-quark interaction term produce $m_{u}=12.5 \mathrm{MeV}$ which is higher than the range $5-9 \mathrm{MeV}$.

The effect of eight-quark interaction on thermodynamic properties are the main interest of our work. The variation of scaled pressure $P / P_{S B}$ with $T / T_{C}$ for four input parameters have been plotted and the results are compared with available lattice data. The inclusion of eight quark interaction decreases the pressure near the transition temperature and increases the pressure at higher temperature. We have also studied the variation of $(\epsilon-3 p) / T^{4}$ with temperature. The six-quark interaction is already in good agreement with $N_{\tau}=6$ lattice data. However, comparison with $N_{\tau}=4$ lattice data gives us the impression that a continuum extrapolation may give a better match to our results for eight-quark interactions.

We discuss the effect of higher order interaction term on the finite density results. We observe that due to the eight-quark interaction quark number density increases above the transition temperature.

The effect of the eight-quark interaction term on the critical end points in the phase diagram is the main issue of our study. From table III we can observe that the eight-quark interaction drives the CEP to a low chemical potential and a high temperature value. Furthermore, our study concludes that the inclusion of eight-quark interaction is essential to limit $\mu_{C} / T_{C}$ below 2.5 as suggested by Lattice calculations.

All these studies lead us to the conclusion that the eight-quark interaction term in the Lagrangian has an interesting phenomenological implication in effective models.

\section{Acknowledgments}

P.D. thanks CSIR for the financial support. A.B. thanks CSIR and UGC for support. A.B. also thanks Brigitte Hiller and Saumen Datta for useful discussions.

[1] Y. Nambu and G. Jona-Lasinio, Phys. Rev. 122, 345 (1961), 124, 246 (1961).

[2] S. P. Klevansky, Rev. Mod. Phys. 64649 (1992).

[3] T.Hatsuda and T.Kunihiro, Phys. Rept. 247, 221 (1994).

[4] T. Kunihiro and T. Hatsuda, Phys. Lett. B 206, 385 (1988).

[5] K. Fukushima, Phys. Lett. B 591, 277 (2004). 
[6] C. Ratti, M. A. Thaler and W. Weise, Phys. Rev. D 73, 014019 (2006).

[7] S. K. Ghosh, T. K. Mukherjee, M. G. Mustafa and R. Ray, Phys. Rev. D 77, 0904024 (2008).

[8] M. Ciminale, R. Gatto, N. D. Ippolito, G. Nardulli and M. Ruggieri, Phys. Rev. D 77, 054023 (2008).

[9] P. Deb, A. Bhattacharyya, S. Datta and S. K. Ghosh, Phys. Rev. C 79, 055208 (2009).

[10] H. Reinhardt and R. Alkofer, Phys. Lett. B 207482 (1988).

[11] E. V. Shuryak, Phys. Rep. 391, 381 (2004).

[12] Yu. A. Simonov, Phys. Lett. B 412371 (1997).

[13] Yu. A. Simonov, Phys. Rev. D 65094018 (2002).

[14] A. A. Osipov, B. Hiller and J. da Providencia, Phys. Lett. B 634, 48 (2006)

[15] A. A. Osipov, B. Hiller, A. H. Blin and J. da Providencia, Annl. Phys. 322, 2021-2054 (2007)

[16] J. Moreira, B. Hiller, A. A. Osipov and A. H. Blin, arXiv:1001.3565 [hep-ph]

[17] K. Kashiwa, H. Kouno, T. Sakaguchi, M. Matsuzaki and M. Yahiro, Phys. Lett. B 647, 446-451 (2007).

[18] K. Kashiwa, H. Kouno, M. Matsuzaki and M. Yahiro, Phys. Lett. B 662, 26-32 (2008).

[19] A. Dumitru and R. D. Pisarski, Nucl. Phys. Proc. Suppl. 106, 483 (2002).

[20] R. Pisarski, Phys. Rev. D 62, 111501(R) (2000).

[21] S. K. Ghosh, T. K. Mukherjee, M. G. Mustafa and R. Ray, Phys. Rev. D 73114007 (2006).

[22] S. Mukherjee, M. G. Mustafa and R. Ray, Phys. Rev. D 75, 094015 (2007).

[23] M. Cheng et al., Phys. Rev. D 77, 014511 (2008).

[24] J. Engels et al., Nucl. Phys. B 469, 419 (1996).

[25] R. Pisarski, Phys. Rev. D 74, 121703 (2006).

[26] Z. Fodor and S. D. Katz, JHEP 0203 (2002) 014; Z. Fodor, S. D. Katz, Prog. Theor. Phys. Suppl. 153 (2004) 86.

[27] S. Ejiri, arXiv:0706.3549] [hep-lat]; S. Ejiri, arXiv:0710.0653 [hep-lat]. 